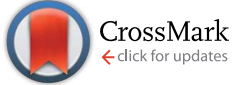

Cite this: RSC Adv., 2016, 6, 27750
Received 1st February 2016

Accepted 9th March 2016

DOI: $10.1039 / c 6 r a 03016 e$

www.rsc.org/advances

\section{Is it possible to substitute hexane with green solvents for extraction of carotenoids? A theoretical versus experimental solubility study}

\author{
E. Yara-Varón, ${ }^{\text {ab }}$ A. S. Fabiano-Tixier, ${ }^{\text {ac }}$ M. Balcells, ${ }^{a}$ R. Canela-Garayoa, ${ }^{a}$ Antoine Bily ${ }^{c}$ \\ and F. Chemat ${ }^{\star a c}$
}

\begin{abstract}
The present study was designed to evaluate five green solvents, i.e. 2-methyltetrahydrofuran (2-MeTHF), dimethyl carbonate (DMC), cyclopentyl methyl ether (CPME), isopropyl alcohol (IPA) and ethyl acetate, for the substitution of $n$-hexane in the extraction of carotenoids from carrots. Initially, solvent selection was made through the theoretical physicochemical solvent properties and solubility results obtained using two simulation programs, Hansen Solubility Parameters (HSPs) and Conductor-like Screening Model for Realistic Solvation (COSMO-RS) which use a statistical thermodynamics approach based on the result of quantum chemical calculation, for comprehension of the dissolving mechanism. On the basis of the HSPs analysis, non-polar or slightly polar solvents were the most suitable solvents for extraction of carotenoids. COSMO-RS analysis showed a higher probability of solubility for all the carotenoids from carrot in CPME, 2-MeTHF and ethyl acetate compared with $n$-hexane. The experimental results using a conventional solid-liquid extraction by maceration showed that the best green solvents were CPME, 2-MeTHF and ethyl acetate in accordance with the predictive results from COSMO-RS. The highest carotenoid content (78.4 mg $100 \mathrm{~g}^{-1}$ DM) was observed in CPME where 66\% was represented by $\beta$-carotene and $34 \%$ was $\alpha$-carotene. These results support the potential of CPME and 2-MeTHF as alternative green solvents for extraction of carotenoids.
\end{abstract}

\section{Introduction}

Nowadays the carotenoids are one of the major groups of natural pigments that find widespread utilization in the food industry. Furthermore, they are also used for medical, cosmetic, and biotechnological purposes. The use of artificial coloring in the modern food industry is common practice, but there is growing concern about their actual or potential effects on human health. This concern has led to an increasing interest and utilization of natural and bioactive products as alternative food colorants. The addition of colorants such as carotenoids to food serves for one or more of the following purposes: (1) to replace color lost during processing, (2) to enhance color already present, (3) to minimize batch-to-batch variations, (4) to color otherwise uncolored food and (5) give nutritional value, since they are bioactive compounds. ${ }^{1}$ It is therefore not surprising that the global market value for carotenoids was estimated in USD\$1.5 billion in 2014 and is projected to reach nearly USD $\$ 1.8$ billion in 2019 , with a compound annual growth

\footnotetext{
${ }^{a}$ Université d'Avignon et des Pays de Vaucluse, INRA, UMR408, GREEN Team Extraction, F-84000 Avignon Cedex, France. E-mail: farid.chemat@univ-avignon.fr; Tel: +330490144465

${ }^{b}$ Department of Chemistry, University of Lleida, Rovira Roure 191, 25198 Lleida, Spain 'Naturex, 250 rue Pierre Bayle, BP 81218, F-84911 Avignon Cedex 9, France
}

rate (CAGR) of $3.9 \%{ }^{2}$ The carotenoid market can be broken down by compound, representing 10 submarkets for $\beta$-carotene, lutein, astaxanthin, capsanthin, annatto, canthaxanthin, lycopene, $\beta$-apo-8-carotenal, zeaxanthin and $\beta$-apo-8-carotenalester. The largest market for individual carotenoids is $\beta$-carotene (USD\$261 million in 2010, projected to grow to USD\$334 million by 2018, CAGR 3.1\%). ${ }^{3}$ This compound holds unique properties as a colorant, physiological antioxidant and provitamin A. This versatility means that it is used across many different segments, which include food, feed, supplements and cosmetics. In the EU, $\beta$-carotene is defined as a food additive (E160a) according to Commission Regulation (EU) 231/2012 and may be chemically synthesized, extracted from plants, or produced by the cultivation of Blakeslea trispora (fungus) or Dunaliella salina (green algae). ${ }^{3}$

Carotenoids are known to be natural pigments responsible for the red, orange and yellow colours of plant leaves, fruits, and flowers, as well as for the color of feathers, crustacean shells, fish flesh and skin, etc. These compounds can be biosynthesized as secondary metabolites by a great number of plants, algae, bacteria and fungi. They are formed by polymerization of isoprene units to an aliphatic or alicyclic structure. The term carotenoid represents a wide range of chemicals, which include two major classes of pigments: firstly, carotenes (e.g., $\alpha$-carotene, $\beta$-carotene, lycopene), which are exclusively 
hydrocarbons, and secondly, xanthophylls (e.g., lutein, zeaxanthin, fucoxanthin and astaxanthin). ${ }^{4}$ Humans cannot synthesize these compounds; therefore, they must be obtained from dietary sources plants being the most important ones. Carotenoids are important compounds to human health, due to their protective effects against serious disorders such as cancer, heart disease and degenerative eye diseases. ${ }^{5}$

Due to their moderate to high hydrophobicity, the extraction of carotenoids from vegetable sources is usually carried out by using organic solvents (e.g., hexane, acetone, dichloromethane, chloroform, ethanol, etc.). However, these solvents are usually volatile organic compounds (VOCs) sourced mainly from nonrenewable resources. They are flammable, volatile, and often toxic and are responsible for environmental pollution and the greenhouse effect. Nowadays, $n$-hexane is one of the most used solvents to extract non-polar carotenoids for its various qualities such as low polarity, ease removal from the products by evaporation, convenient boiling point (high enough to limit losses during extraction but sufficiently low to limit heat consumption during its recovery) and stability. ${ }^{6}$ However, solvents of petrochemical origin as $n$-hexane are now strictly regulated by European Directives and Registration, Evaluation, Authorisation and Restriction of Chemicals (REACH). Therefore, industries are forced to turn to use more environmentally friendly alternatives as green solvents, some of them produced from renewable resources as biomass (wood, starch, vegetable oils or fruits). Green solvents have to satisfy the twelve principles of Green Chemistry developed by Anastas and Warner. ${ }^{7}$ Thus, according to Estévez ${ }^{8}$ green solvents are defined as solvents which has to bear a number of environment, health and safety (EHS) characteristics that differentiate it from existing hazardous solvents. Hence, the adjective "green" is used to describe different types of solvents including the ones that are produced from biomass feedstock (bio-based solvents) and ecofriendly petrochemical-based solvents that are non-toxic and/or biodegradable. ${ }^{9}$ The importance and the potential of green solvents that could be alternatively used instead of the petrochemical ones has been widely studied. Examples of that are the extractions of fat and oils from rapeseed using MeTHF instead of hexane, ${ }^{6}$ extraction of oil from microalgae using D-limonene instead of hexan $\mathrm{e}^{\mathbf{1 0}}$ and extraction of $\alpha$-mangostin from Garcinia mangostana L. using as alternative solvents DMC, MeTHF and ethyl acetate. ${ }^{\mathbf{1 1}}$

In this paper, the selection of the most appropriate solvents to extract carotenoids from carrot (Daucus carota) was initially carried out using two predictive computational programs (Fig. 1), i.e. the Hansen solubility parameters (HSPs) and a conductor like screening model for realistic solvation (COSMO-RS) which are being used to predict the most suitable solvents for extraction of natural products. ${ }^{12-14}$ Finally, after the completely in silico approach and considering the physicochemical properties we selected five alternative bio-solvents $(n$ hexane, 2-MeTHF, DMC, CPME, IPA and ethyl acetate) to experimentally evaluate their ability compared with $n$-hexane to extract carotenoids from carrots. We performed appropriate comparisons in terms of solubility, carotenoid extraction yields and economic versus ecological parameters.

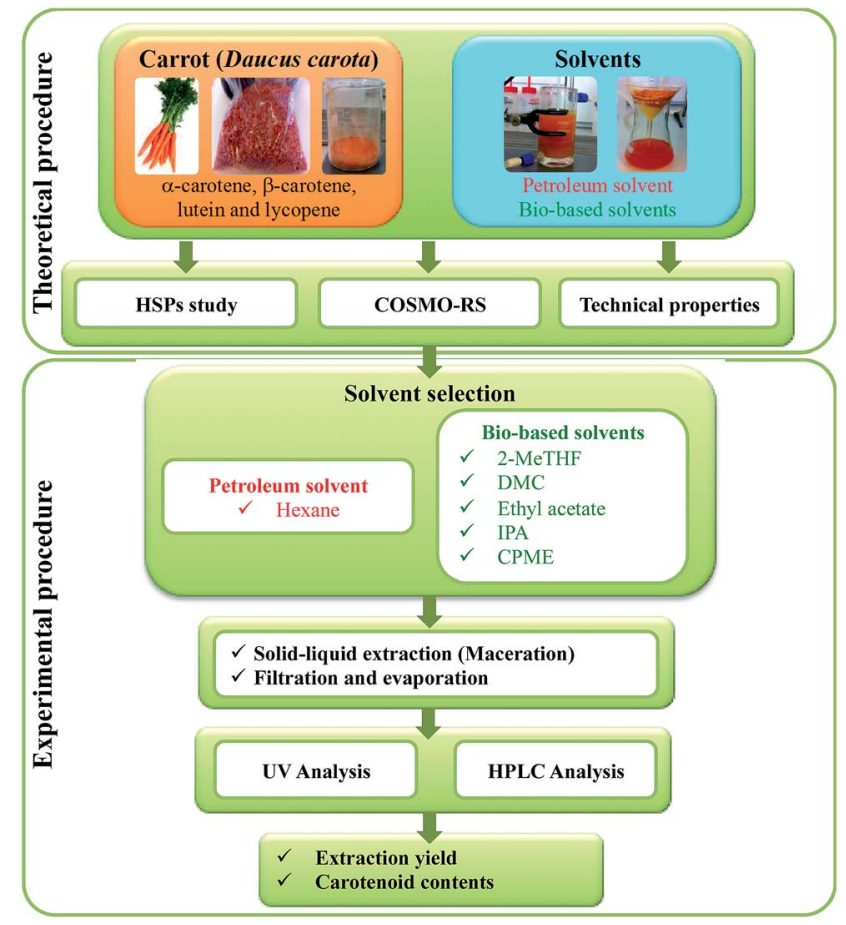

Fig. 1 Experimental design and diagram for extraction of carotenoids from carrots (Daucus carota). 2-Methyltetrahydrofuran (2-MeTHF), dimethyl carbonate (DMC), isopropyl alcohol (IPA), cyclopentyl methyl ether (CPME), ultra violet spectroscopy analysis (UV), high performance liquid chromatography (HPLC), Hansen solubility parameters (HSPs), conductor like screening model for realistic solvation (COSMO-RS).

\section{Materials and methods}

\section{Plants material}

Carrots (Daucus carota) cut in small pieces and dried were provided by Naturex Company (Avignon, France). The dried carrot pieces were kept in a well-closed container and protected from light until further use.

\section{Reagents}

n-Hexane (HPLC grade), 2-methyltetrahydrofuran (2-MeTHF) (purity 99\%), ethyl acetate (purity 99\%) and isopropyl alcohol (IPA) (purity 99.9\%) were supplied by VWR International (Darmstadt, Germany). Dimethyl carbonate (DMC) (purity 99\%) was from Alfa Aesar, Germany. Cyclopentyl methyl ether (CPME) (reagent plus 99.99\%), $\beta$-carotene and lutein standards (9599\%) were provided by Sigma Aldrich, Germany.

\section{Computational methods}

Solubility parameters of solvents have been studied by means of Hansen Solubility Parameters (HSP) theoretical prediction and COSMO-RS.

\section{Hansen solubility parameters}

Solubility parameters for predicting the solubility of a solute were proposed by Hansen. ${ }^{15}$ HSP provides a convenient and 
efficient way to characterize solute-solvent interactions according to the classical "like dissolves like" rule. HSP are based on the concept that the total cohesive energy density is approximated by the sum of the energy densities required to overcome atomic dispersion forces $\left(\delta_{\mathrm{d}}^{2}\right)$, molecular polar forces arising from dipole moments $\left(\delta_{\mathrm{p}}{ }^{2}\right)$ and hydrogen-bonds between molecules $\left(\delta_{\mathrm{h}}{ }^{2}\right)$, as given in the following equation.

$$
\delta_{\text {total }}^{2}=\delta_{\mathrm{d}}^{2}+\delta_{\mathrm{p}}^{2}+\delta_{\mathrm{h}}^{2}
$$

where $\delta_{\text {total }}$ is the Hansen total solubility parameter, which consists of three HSP in terms of dispersion $\left(\delta_{\mathrm{d}}\right)$, polar $\left(\delta_{\mathrm{p}}\right)$ and hydrogen-bonding $\left(\delta_{\mathrm{h}}\right)$. For HSP solvent optimization, a simple composite affinity parameter, the relative energy difference (RED) number, has been calculated using eqn (2) to determine the solubility between solvent and solute.

$$
\mathrm{RED}=R_{\mathrm{a}} / R_{\mathrm{O}}
$$

where $R_{\mathrm{O}}$ is the radius of a Hansen solubility sphere, and $R_{\mathrm{a}}$ is the distance of a solvent from the centre of the Hansen solubility sphere, given by eqn (3):

$$
R_{\mathrm{a}}^{2}=4\left(\delta_{\mathrm{d}} \mathrm{A}-\delta_{\mathrm{d}} \mathrm{B}\right)^{2}+\left(\delta_{\mathrm{p}} \mathrm{A}-\delta_{\mathrm{p}} \mathrm{B}\right)^{2}+\left(\delta_{\mathrm{h}} \mathrm{A}-\delta_{\mathrm{h}} \mathrm{B}\right)^{2}
$$

where A refers to the solute and B refers to the solvent. The factor 4 in eqn (3), based on Prigogine's Corresponding States Theory, has proved to effectively expand the dimensions in order to give spherical plots. The smaller $R_{\mathrm{a}}$ is the greater affinity between solute and solvent. It means that good potential solvents have an RED of less than 1 (the compound has similar properties and will dissolve), while medium and poor solvents have RED values of from 1 to 3 and more than 3, respectively.

The chemical structures of the solvents and solutes (Fig. 2) discussed in this article could be mutually transformed by JChemPaint version 3.3 (GitHub Pages, San Francisco, CA, USA) to their simplified molecular input line entry syntax (SMILES) notations, which were subsequently used to calculate the solubility parameters of various solvents and carotenoids. These solubility parameters were further modelled to a threedimensional HSP sphere for better visualizing the solute/ solvent interactions (HSPiP Version 4.0, Hansen-Solubility, Hørsholm, Denmark).

\section{COSMO-RS}

The Conductor-like Screening Model for Real Solvents (COSMORS) uses a statistical thermodynamics approach based on the result of quantum chemical calculations for an understanding of the dissolving mechanism. COSMO-RS combines quantum chemical considerations (COSMO) and statistical thermodynamics (RS) to determine and predict the thermodynamic properties without experimental data. The COSMO-RS developed by Klamt ${ }^{16}$ is known as a powerful method for molecular description and solvent screening based on a quantumchemical approach.

COSMO-RS is a two-step procedure including microscopic and macroscopic steps. In the first step, the COSMO model is applied to simulate a virtual conductor environment for the molecule of interest. The molecule is embedded into a virtual conductor (Fig. 3a). In such an environment the molecule induced a polarization charge density on its surface (Fig. 3b). Thus, during the quantum calculation self-consistency algorithm the solute molecule is converged to its energetically optimal state in the conductor with respect to its electron density and geometry. The standard quantum chemical methods, triple zeta valence polarized basis set (TZVP) was used in this study.

The second step used the statistical thermodynamic calculation. This polarization charge density was used for the quantification of the interaction energy of the pair-wise interacting surface segments with regard the most important molecular interaction modes, i.e. electrostatics and hydrogen bonding. The 3D distribution of the polarization charges on the surface of each molecule was converted into a surface composition function ( $\sigma$-profile). Such $\sigma$-profile provided detailed information about the molecular polarity distribution (Fig. 3c). The thermodynamics of the molecular interactions that were based on the obtained s-profile was then used to calculate the chemical potential of the surface segment ( $\sigma$-potential) as shown in Fig. 3d using COSMOthermX program (version C30 release 13.01).

The $\sigma$-potential described the likeliness of the compound being able to interact with the solvents with polarity and hydrogen bonds (Fig. 3d). The part of the negative charge of the molecule was located on the right side (acceptor hydrogen bonds) with positive $\sigma$ values while the part of the positive charged was located on the left side (donor hydrogen bonds) with negative $\sigma$ values. Generally, the region $\sigma \pm 0.01$ e $\mathrm{A}^{-2}$ was considered to be non-polar or weakly polar. The $\sigma$-profile and the $\sigma$-potential were used for interpreting the affinity of the solvent for surface polarity, to understand the interaction between the compound and solvent in mixed state and to estimate the thermodynamic properties of the system.

In addition, the COSMOthermX also calculated the relative solubility between the solid compound and the liquid solvent in terms of the logarithm of the solubility in mole fractions $\left(\log _{10}\left(x_{\text {solub }}\right)\right)$. The logarithm of the best solubility was set to 0 and all other solvents were given relative to the best solvent. Also, the logarithm was transformed into probability of solubility and was expressed in percentage. The calculation for carotenoids ( $\beta$-carotene, $\alpha$-carotene, lutein and lycopene) and solvents was performed at $65{ }^{\circ} \mathrm{C}$.

\section{Carotenoid extraction}

The carotenoids were separately extracted by solid-liquid extraction with $n$-hexane, 2-MeTHF, ethyl acetate, DMC, CPME and IPA $(125 \mathrm{~mL})$. Immediately before to extraction the dried carrot pieces were ground into a fine powder using a grinder. An amount of $30 \mathrm{~g}$ of the powder was added to the jacketed reactor with the solvent at $65{ }^{\circ} \mathrm{C}$. The mixture was mechanically stirred and macerated for $1 \mathrm{~h}$ in the dark. Afterwards, the mixture was filtered through cotton, while the solid residue was 


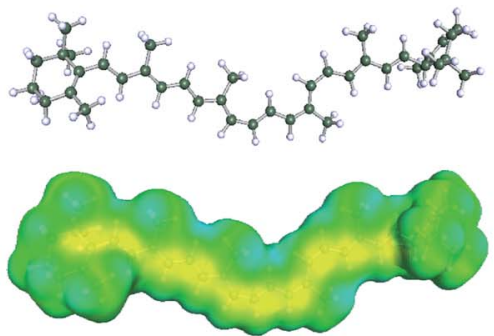

$\alpha$-Carotene

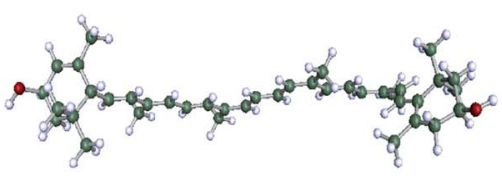

Lutein
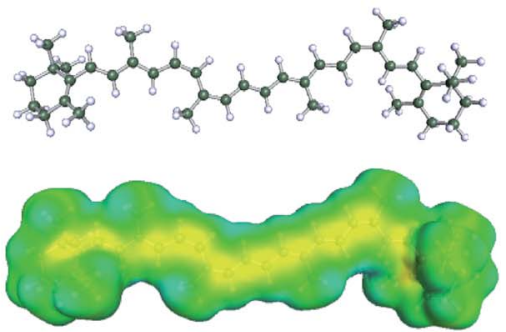

$\beta$-Carotene
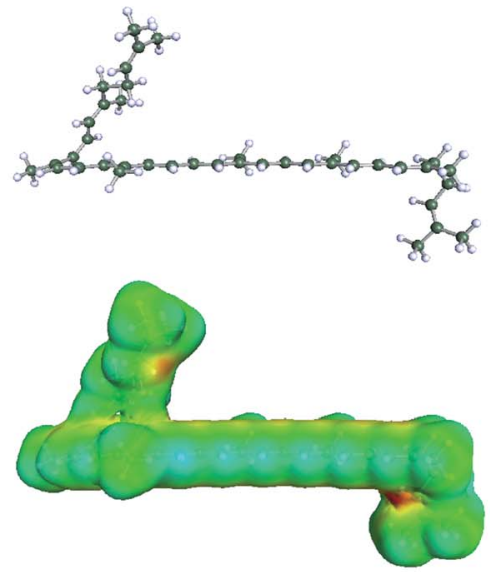

Lycopene

Solvents

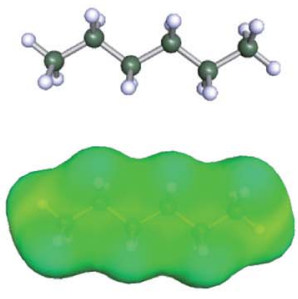

Hexane
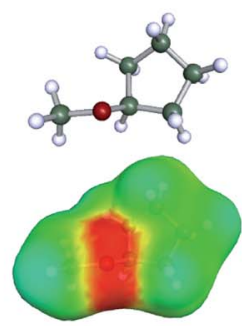

CPME

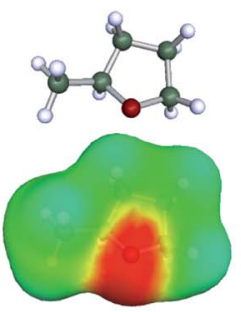

2-MeTHF
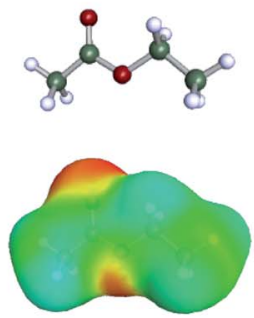

Ethyl acetate

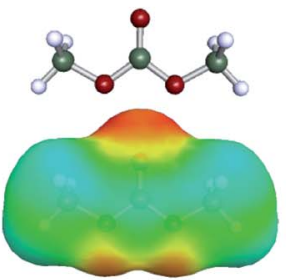

DMC

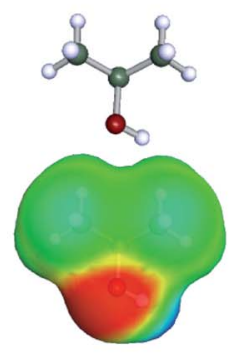

IPA

Fig. 2 Structures and $\alpha$-surfaces of carotenoids and solvents used in experimental study.

washed two times with fresh solvent. After extraction, the solvent was evaporated under vacuum in a rotary evaporator. The residue was dissolved with $n$-hexane, transferred to a smaller flask and dried with $\mathrm{N}_{2}$ stream. The samples were stored at $-20{ }^{\circ} \mathrm{C}$ until analyzed. All experiments were carried out in triplicates. 


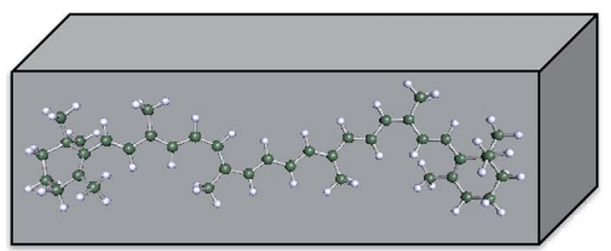

a) $\beta$-carotene

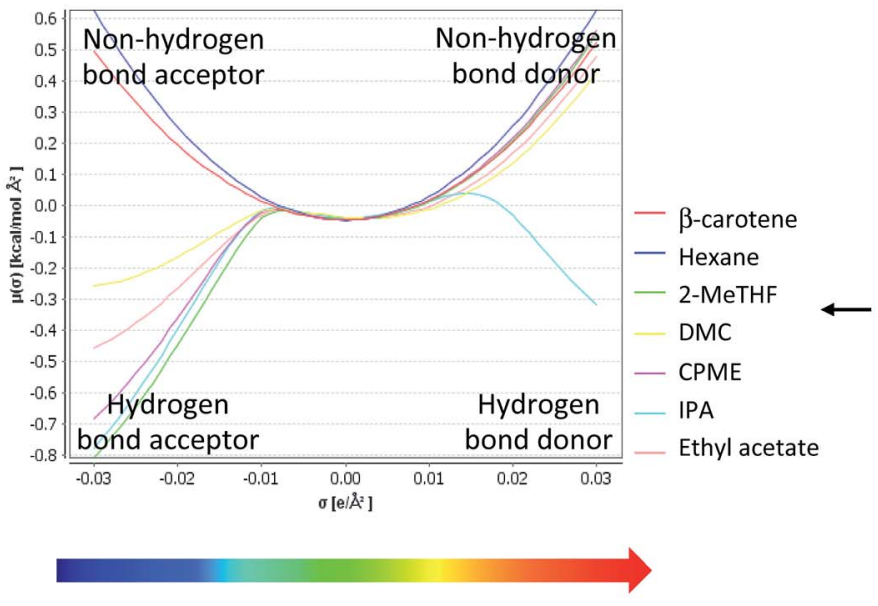

d) $\sigma$-potential

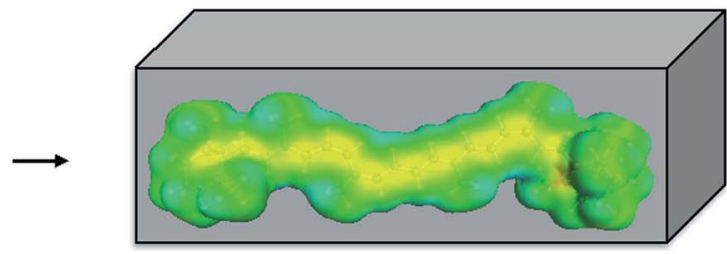

b) $\sigma$-surface
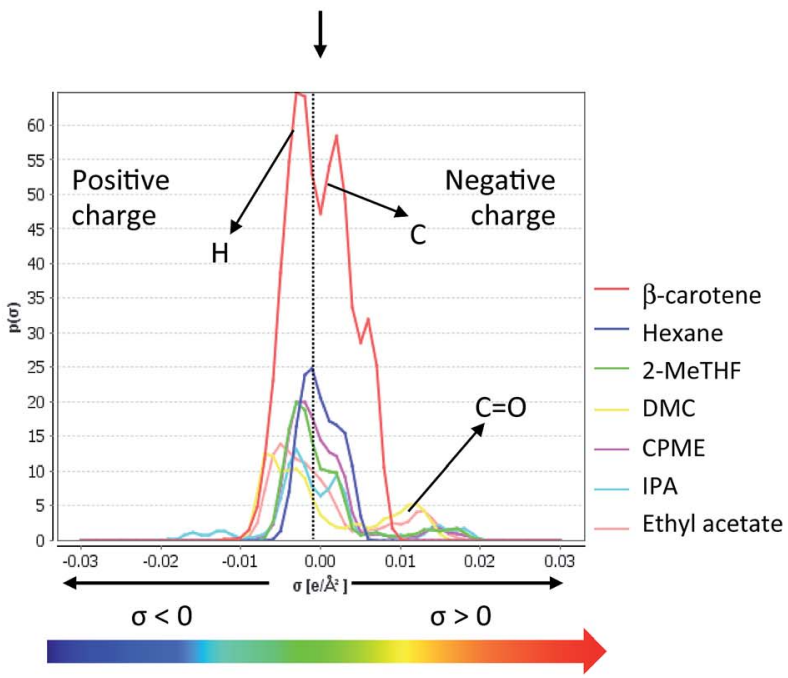

c) б-profile

Fig. 3 Step calculation with COSMO-RS: (a) molecule emerged; (b) $\sigma$-surface; (c) energies of local surface interactions between $\sigma$-profiles of $\beta$ carotene and solvents; (d) $\sigma$-potentials of $\beta$-carotene and solvents.

\section{Ultra violet (UV) spectroscopy analysis}

The carotenoid content was measured spectrophotometrically (Biochrom Libra S22 UV/Vis Spectrophotometer) in a $1 \mathrm{~cm}$ optical path-length quartz cell at $450 \mathrm{~nm}$ for $\beta$-carotene in each extract against the $n$-hexane used as a blank. The Beer-Lambert law was used to determine the carotenoid concentration in each extract. The following equation was used to calculate the carotenoid concentration, ${ }^{17} C\left(\mathrm{mg} \mathrm{L}^{-1}\right)$ :

$$
C=\frac{A_{\lambda_{\max } \times 10^{4}}}{A_{1 \mathrm{~cm}}^{1 \%}}
$$

where $A_{\lambda_{\max }}$ is the absorbance of the extract at $\lambda_{\max }$ and $A_{1 \mathrm{~cm}}^{1 \%}$ is the absorption coefficient (absorbance at $1 \mathrm{~cm}$ a given wavelength of a $1 \%$ solution in a spectrophotometer cuvette with a 1 $\mathrm{cm}$ light path) of $\beta$-carotene in the respective solvent. The absorption coefficient was 2592 in $n$-hexane at $450 \mathrm{~nm}$. Finally the yield of carotenoids in each extract was calculated and expressed as $\mathrm{mg}$ ( $\beta$-carotene) $100 \mathrm{~g}^{-1}$ of dry matter.

\section{Quantitative analysis of carotenoids by HPLC}

HPLC analysis was carried out using a HPLC Agilent 1100 equipped with a UV-VIS detector (DAD). Separation was achieved at $25^{\circ} \mathrm{C}$ on a C18 column $(150 \times 3.0 \mathrm{~mm}$ and $3 \mu \mathrm{m}$ ID $)$. The mobile phase consisted of acetonitrile/methanol (with $0.6 \%$ ammonium acetate)/dichloromethane $(77 / 20 / 3, \mathrm{v} / \mathrm{v} / \mathrm{v})$, and was pumped at a flow rate of $1.4 \mathrm{~mL} \mathrm{~min}^{-1}$. The sample injection volume was $10 \mu \mathrm{L}$ and the quantitative detection was at a wavelength of $464 \mathrm{~nm}$. The calibration curves of carotenoids were established using standard carotenoids (concentration range was $5,10,25,50$ and $100 \mathrm{mg} \mathrm{L}^{-1}$ ). Each calibration point was carried out in triplicate. The identification of major carotenoids in carrot extracts was carried out by comparing the retention times and absorption spectra.

\section{Results and discussion}

This study has been performed by theoretical procedure using two computational predictive methods (HSPs and COSMO-RS), technical properties of the solvents and via experimentation. The comparison was made in terms of amount of carotenoids in each extract and taking into account technical and economical parameters of the solvents used in the carotenoid extraction.

\section{Solute-solvent solubility by HSPs}

Initially, several solvents with different polarity were selected to carry out the simulation by HSPs. The solubility of the carotenoids in each solvent used in the theoretical study was compared with $n$-hexane, which is one of the most used solvents for extraction of these compounds. The software allows the assessment of the relative energy difference (RED), which estimates the capacity of a solvent to dissolve solutes. Table 1 shows the RED calculated for every selected solvent with carotenoids. The RED values $<1$ represent a good solubility which means that 
Table 1 Relative energy difference (RED) values to $n$-hexane for HSP assisted selection of solvents for the extraction of carotenoids ${ }^{a}$

\begin{tabular}{|c|c|c|c|c|c|c|c|}
\hline \multirow{2}{*}{ Solvent } & \multirow{2}{*}{$\delta_{\mathrm{d}}$} & \multirow{2}{*}{$\delta_{\mathrm{p}}$} & \multirow{2}{*}{$\delta_{\mathrm{h}}$} & \multicolumn{4}{|c|}{ Red } \\
\hline & & & & $\alpha$-Carotene & $\beta$-Carotene & Lutein & Lycopene \\
\hline$n$-Hexane & 14.90 & 0.00 & 0.00 & 1.31 & 1.34 & 1.97 & 1.27 \\
\hline$\alpha$-Pinene & 17.00 & 1.30 & 2.00 & 0.40 & 0.25 & 0.88 & 0.37 \\
\hline$\beta$-Pinene & 16.30 & 1.10 & 1.90 & 0.62 & 0.56 & 1.10 & 0.57 \\
\hline D-Limonene & 17.20 & 1.80 & 4.30 & 0.84 & 0.70 & 0.36 & 0.79 \\
\hline$p$-Cymene & 18.50 & 2.60 & 1.90 & 0.86 & 0.71 & 0.91 & 0.89 \\
\hline$\beta$-Myrcene & 16.00 & 1.60 & 2.20 & 0.82 & 0.74 & 1.16 & 0.77 \\
\hline Ethyl oleate & 16.20 & 2.30 & 2.90 & 0.90 & 0.77 & 0.99 & 0.85 \\
\hline Isopropyl palmitate & 15.90 & 2.20 & 2.50 & 0.96 & 0.85 & 1.16 & 0.91 \\
\hline Ethyl laurate & 16.00 & 3.10 & 3.50 & 1.16 & 1.01 & 1.05 & 1.11 \\
\hline CPME & 16.70 & 4.30 & 4.30 & 1.14 & 1.33 & 0.89 & 1.29 \\
\hline 2-MeTHF & 16.40 & 4.80 & 4.60 & 1.51 & 1.33 & 1.07 & 1.47 \\
\hline Ethyl acetate & 15.80 & 5.30 & 7.20 & 2.10 & 1.95 & 1.46 & 2.05 \\
\hline Methyl acetate & 15.50 & 7.20 & 7.60 & 2.54 & 2.37 & 1.92 & 2.50 \\
\hline DMC & 15.90 & 8.70 & 7.10 & 2.69 & 2.51 & 2.07 & 2.65 \\
\hline IPA & 15.80 & 6.60 & 14.30 & 3.69 & 3.56 & 2.80 & 3.63 \\
\hline 1-Butanol & 16.00 & 5.70 & 15.80 & 3.91 & 3.80 & 3.00 & 3.86 \\
\hline Ethanol & 15.80 & 8.80 & 19.40 & 5.05 & 4.92 & 4.13 & 5.00 \\
\hline Methanol & 14.70 & 12.30 & 22.30 & 6.19 & 6.05 & 5.30 & 6.14 \\
\hline Water & 15.50 & 16.00 & 42.30 & 11.00 & 10.88 & 10.04 & 10.95 \\
\hline
\end{tabular}

${ }^{a}$ Gray color: reference; green color: better solvent than reference $(<1)$; yellow color: equivalent or slightly worse solvent than reference $(1-3)$; red color: worse solvent than reference $(>3)$.

$n$-hexane is not the best solvent, from a theoretical perspective, for the extraction of the carotenoids evaluated in this work. According to the results, terpenes ( $\alpha$-pinene, $\beta$-pinene, D-limonene, $p$-cymene and $\beta$-myrcene), ethyl oleate and isopropyl palmitate are better solvents for the extraction of carotenoids than $n$-hexane. Other solvents (ethyl laurate, CPME, 2-MeTHF, ethyl acetate, methyl acetate and DMC) are theoretically equivalent or some of them slightly worse than the reference. Finally, polar solvents (isopropyl alcohol, 1-butanol, ethanol, methanol and water) were worse than $n$-hexane in the HSPs theoretical study. This can be explained by the difference in the polarity of these solvents regarding to the solutes.

\section{COSMO-RS prediction}

The COSMO-RS simulation was also conducted in order to determine the potential of various solvents for the extraction of carotenoids. The software integrates a quantum chemistry approach that permits the calculation of various properties such as the relative solubility of a compound in several solvents. It means, that the analysis of the $\sigma$-profile and $\sigma$-potential of the components in the mixture (carotenoids and solvents) gives some important information about the molecules that can be used to predict possible interactions in the fluid phase. For example, Fig. $3 c$ shows the $\sigma$-profile of six solvents ( $n$-hexane, 2MeTHF, DMC, CPME, IPA and ethyl acetate) and the main carotenoid from carrot: $\beta$-carotene. Generally, the region $\pm 1 \times$ $10^{-2} \mathrm{e} \mathrm{A}^{-2}$ is considered as non-polar or weakly polar. The $\sigma$ profile of $\beta$-carotene shows two peaks; the first peak resulted from the polarized hydrogen atoms and the second peak at a $\sigma$ approximately of $0.005 \mathrm{e} \mathrm{A}^{-2}$ resulted from the carbon atoms (Fig. 3c). Therefore, the $\sigma$-potential of $\beta$-carotene is symmetric (Fig. 3d). The $\sigma$-profile of the $n$-hexane also shows two peaks, resulting from the hydrogen atoms on the negative side and from the carbons atoms on the positive side. As a consequence, the $\sigma$-potential of $n$-hexane is similar to the parabola with center at $\sigma=0$, a characteristic of a non-polar solvents and similar to the $\sigma$-profile and $\sigma$-potential of the $\beta$-carotene.

Table 2 shows the solubility of the main carotenoids from carrot in the different solvents used in this study expressed in $\log _{10}\left(x_{\text {solub }}\right)$ (best solubility is set to 0$)$ and percentage of probability of solubility for a better understanding of the results. It can be noted that the prediction was carried at $65^{\circ} \mathrm{C}$, which is the temperature of extraction under industrial conditions. According to the rule "like dissolves like" $n$-hexane and the other non-polar or weakly polar solvents evaluated in this study show high probability of solubility (60-100\%) for $\beta$-carotene, $\alpha$ carotene and lycopene. However, those solvents presented low $(0-20 \%)$ or medium (20-60\%) solubility for lutein, that can be due to the hydroxyl groups of lutein that give higher polarity to the molecule. On the contrary, polar solvents exhibited better 
Table 2 COSMO-RS: relative solubility $\left(\log _{10}\left(x_{\text {solub }}\right)\right)$ and probability of solubility of carotenoids in several solvents at $65^{\circ} \mathrm{C}^{a}$

\begin{tabular}{|c|c|c|c|c|c|c|c|c|}
\hline \multirow[b]{2}{*}{ Solvent } & \multicolumn{2}{|c|}{$\alpha$-Carotene } & \multicolumn{2}{|c|}{$\beta$-Carotene } & \multicolumn{2}{|c|}{ Lutein } & \multicolumn{2}{|c|}{ Lycopene } \\
\hline & $\log _{10}\left(x_{\text {solub }}\right)$ & $\begin{array}{c}\text { Probability } \\
\text { (\%) }\end{array}$ & $\log _{10}\left(x_{\text {solub }}\right)$ & Probability (\%) & $\log _{10}\left(x_{\text {solub }}\right)$ & $\begin{array}{c}\text { Probability } \\
(\%)\end{array}$ & $\log _{10}\left(x_{\text {solub }}\right)$ & Probability (\%) \\
\hline$n$-Hexane & 0.0000 & 100.0 & 0.0000 & 100.0 & -1.3680 & 4.3 & -0.1340 & 73.4 \\
\hline$\alpha$-Pinene & 0.0000 & 100.0 & 0.0000 & 100.0 & -0.9551 & 11.1 & 0.0000 & 100.0 \\
\hline$\beta$-Pinene & 0.0000 & 100.0 & 0.0000 & 100.0 & -0.8087 & 15.5 & 0.0000 & 100.0 \\
\hline D-Limonene & 0.0000 & 100.0 & 0.0000 & 100.0 & -0.6989 & 20.0 & 0.0000 & 100.0 \\
\hline p-Cymene & 0.0000 & 100.0 & 0.0000 & 100.0 & -0.6193 & 24.0 & 0.0000 & 100.0 \\
\hline$\beta$-Myrcene & 0.0000 & 100.0 & 0.0000 & 100.0 & -0.9270 & 11.8 & 0.0000 & 100.0 \\
\hline Ethyl oleate & 0.0000 & 100.0 & 0.0000 & 100.0 & -0.3364 & 46.1 & -0.0494 & 89.2 \\
\hline Isopropyl palmitate & -0.0261 & 94.2 & -0.0305 & 93.2 & -0.4138 & 38.5 & -0.1142 & 76.9 \\
\hline Ethyl laurate & 0.0000 & 100.0 & 0.0000 & 100.0 & -0.0854 & 82.1 & 0.0000 & 100.0 \\
\hline CPME & 0.0000 & 100.0 & 0.0000 & 100.0 & 0.0000 & 100.0 & 0.0000 & 100.0 \\
\hline 2-MeTHF & 0.0000 & 100.0 & 0.0000 & 100.0 & 0.0000 & 100.0 & 0.0000 & 100.0 \\
\hline Ethyl acetate & 0.0000 & 100.0 & 0.0000 & 100.0 & 0.0000 & 100.0 & 0.0000 & 100.0 \\
\hline Methyl acetate & 0.0000 & 100.0 & 0.0000 & 100.0 & 0.0000 & 100.0 & 0.0000 & 100.0 \\
\hline DMC & -0.3723 & 42.4 & -0.3598 & 43.7 & 0.0000 & 100.0 & -0.0163 & 96.3 \\
\hline IPA & -1.1490 & 7.1 & -1.1494 & 7.1 & 0.0000 & 100.0 & -1.0961 & 8.0 \\
\hline 1-Butanol & -1.1183 & 7.6 & -1.1214 & 7.5 & 0.0000 & 100.0 & -1.1122 & 7.7 \\
\hline Ethanol & -1.6013 & 2.5 & -1.5994 & 2.5 & 0.0000 & 100.0 & -1.4881 & 3.2 \\
\hline Methanol & -2.5505 & 0.3 & -2.5449 & 0.3 & -0.4357 & 36.7 & -2.3451 & 0.4 \\
\hline Water & -15.5207 & 0.0 & -15.5571 & 0.0 & -11.5261 & 0.0 & -15.3435 & 0.0 \\
\hline
\end{tabular}

${ }^{a}$ Green color: high probability of solubility (60-100\%); yellow color: medium probability of solubility (20-60\%); red color: low probability of solubility $(0-20 \%)$.

solubility for lutein than for the other carotenoids. The best solvents to extract the main carotenoids from carrot compared with $n$-hexane were CPME, 2-MeTHF and ethyl acetate that exhibited a $100 \%$ probability of solubility for the four carotenoids.

Nevertheless, parameters other than solubility were considered (Table 3). In fact, data such as flash point, viscosity, boiling point and energy required for solvent evaporation as well as $\log P$, toxicity category or resource, are physicochemical and technical properties of the solvent that are important for the solvation of specific compounds but also for the implementation of the process at different scales. Therefore, taking in consideration the theoretical results obtained by the two computational predictive methods and the parameters related to energy efficiency shown in the Table 3 (such as energy for solvent evaporation which is dependent on boiling point and has to be as lower as possible ${ }^{6}$ and toxicities) we decided to perform the experimental study using five green solvents (2MeTHF, DMC, CPME, IPA and ethyl acetate) which can have potential to replace the $n$-hexane.

\section{Total carotenoids yield and composition of the extracts}

The experimental extraction solid-liquid of carotenoids from dry carrot using six different solvents was performed by maceration. The results presented in the Table 4 concern the total carotenoids yield (expressed in $\mathrm{mg} 100 \mathrm{~g}^{-1}$ of dry vegetable matter, DM) and the percentage of individual carotenoids by HPLC analysis. All the green solvents used were capable of extracting $\alpha$-carotene and $\beta$-carotene, but lycopene and lutein were not detected in quantifiable amount (only traces were observed). The yield of carotenoids extraction was in the following order: CPME $>2$-MeTHF $>n$-hexane $>$ ethyl acetate $>$
IPA > DMC. The carotenoids extracted with each solvent were different due to the difference in the polarity and solubility between carotenoids and the solvents. The highest carotenoid content (78.4 mg $100 \mathrm{~g}^{-1} \mathrm{DM}$ ) was observed in CPME where $66 \%$ was represented by $\beta$-carotene and $34 \%$ was $\alpha$-carotene. The carotenoid content extracted with CPME was $40 \%$ higher than that obtained with $n$-hexane. Moreover, HPLC analysis of all extracts showed similar relative percentages of $\alpha$-carotene and $\beta$-carotene and it did not reveal any peaks that would indicate isomerization or degradation. According to Moity et al. ${ }^{9}$ CPME is a diprotic dipolar solvent and belong to the cluster III in the COSMO-RS classification. That means that compounds with alkane chemical structure such as carotenes can be easily dissolved in that kind of solvent. Although CPME was the best solvent to extract carotenoids, we have not found any reference in the literature where this solvent has previously been used for this purpose. CPME has been found as a low toxic alternative solvent and has many preferable characteristics for Green Chemistry; it is used in organic synthesis, especially in process chemistry research, in the pharmaceutical industry as well as in the electronic and fragrance industries. ${ }^{\mathbf{1 8 , 1 9}}$ The second solvent that extracted more carotenoids was the 2MeTHF (65.8 mg $100 \mathrm{~g}^{-1} \mathrm{DM}$ ), which represents $18 \%$ more extracted carotenes when compared to $n$-hexane. This result is similar to that published by Sicaire et al. ${ }^{\mathbf{2 0}}$ They found that the starting accessibility and the effective diffusivity of 2-MeTHF were higher compared with $n$-hexane. This means that the washing step with 2-MeTHF permits to solvate a higher amount of carotenoids at the surface of the matter and the extraction is faster with 2 -MeTHF than with $n$-hexane. Finally, they concluded that 2-MeTHF allowed to extract $23 \%$ more carotenes than $n$-hexane. 


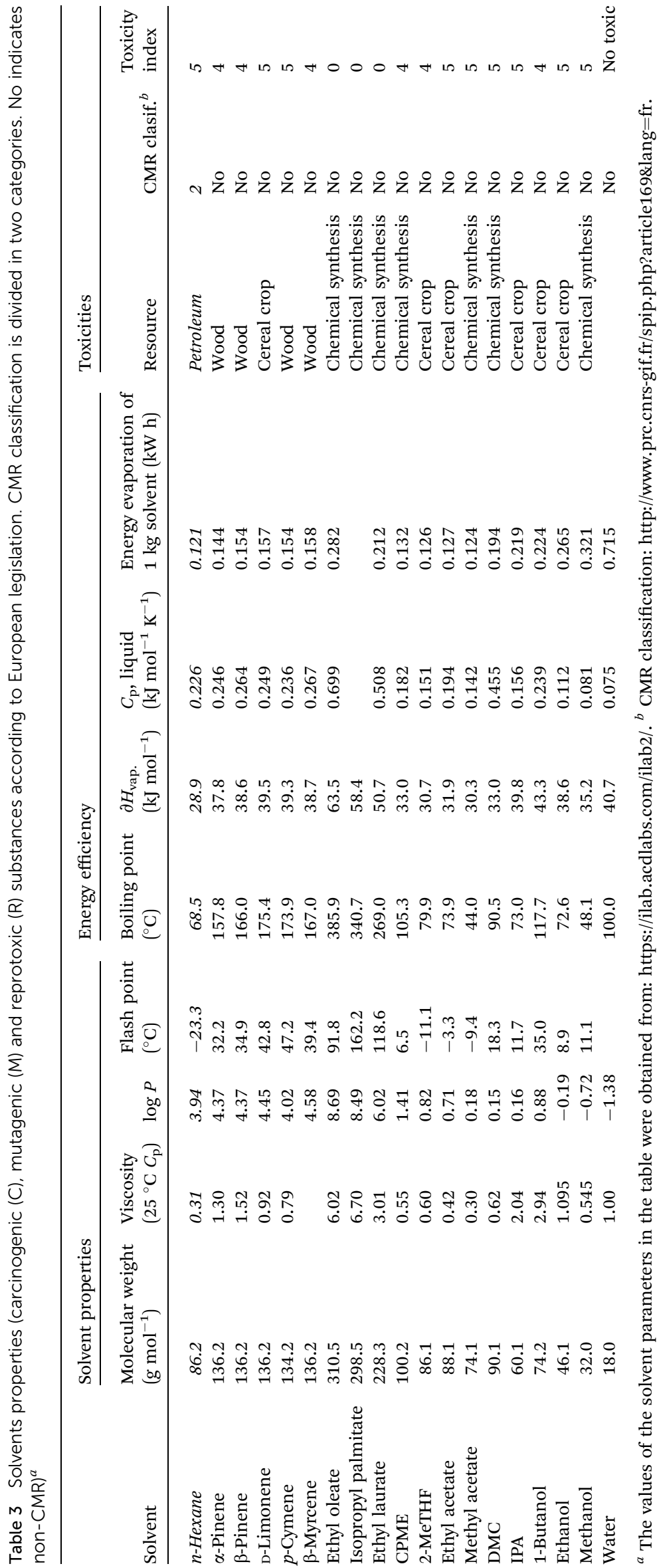


Table 4 Total carotenoid yield (CY), CY compared to maximum carotenoids content in carrots (\%) and percentage of HPLC separated carotenoids from carrot extracted by maceration with different solvents, at $65^{\circ} \mathrm{C}$ for $1 \mathrm{~h}^{b}$

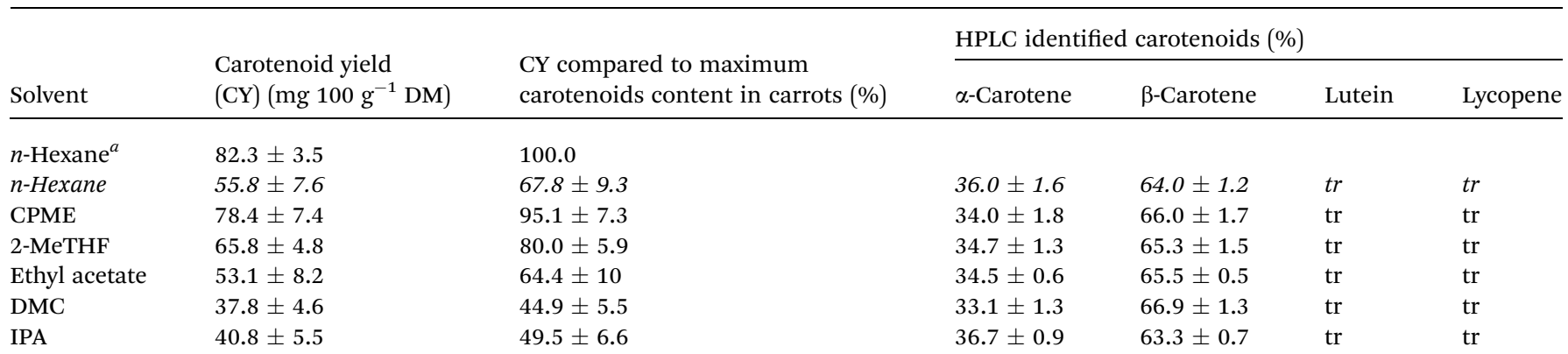

${ }^{a}$ Maximum carotenoids content in carrot ( $n$-hexane at boiling point for $2 \mathrm{~h}$, three cycles). ${ }^{b}$ Values are means $\pm \operatorname{SD}(n=3)$. DM, dry vegetable matter. tr, traces.

To perform a better comparison of the amount of carotenoids obtained by every solvent used in this study, we determined the maximum content of carotenoids in carrots through the extraction with $n$-hexane at boiling point for three extraction cycles (until not color was observed in the solvent). Table 4 shows that the maximum carotenoid content in carrots was 82.3 $\mathrm{mg} 100 \mathrm{~g}^{-1} \mathrm{DM}$. These results are in agreement with that reported by Fikselová, ${ }^{21}$ who described that carrots are one of the best sources of carotenes and its carotene content ranges from 60-120 mg $100 \mathrm{~g}^{-1}$ of DM. Furthermore, $\beta$-carotene constitutes the main part of carrot carotenoids (60-80\%), the fraction of $\alpha$ carotene is $10-40 \%$, and the fraction of lutein does not exceed $1-5 \% .{ }^{22}$ However, there are variations in the composition of carotenoids in vegetables due to factors such as variety, stage of maturity, geographic origin, farming practices and climate or season. ${ }^{\mathbf{2 3 2 4}}$ The percentage of carotenoid extracted by CPME and 2-MeTHF were 95.1 and $80 \%$ of the maximum carotenoid content in carrots. This is 27.3 and $12 \%$ respectively, higher than $n$-hexane. So, these green solvents are potential alternative solvents to $n$-hexane for the extraction of carotenoids from carrots.

\section{Ecological versus economical evaluation}

In recent years, the main objective in extraction of natural products has been set to develop greener and more economically competitive processes for the efficient extraction of natural substances with potential application in the food, cosmetic or agrochemical industries. In this context, we evaluated under ecological and economical terms, the different solvents that we used in this study. Table 5 shows various parameters that allowed the comparison of the green solvents in front of $n$ hexane for the carotenes extraction. First of all, some of the green solvents are prepared by chemical synthesis and others by biochemical transformations of agro-synthons, i.e. defined molecules obtained from the biomass feedstock. ${ }^{9}$ In contrast $n$ hexane is obtained from petroleum and it is classified as a hazardous product for the environment and health. The energy necessary to evaporate $1 \mathrm{~kg}$ of solvent $(E)$ was calculated according to Sicaire et $a l .{ }^{6}$ using the specific heat, latent heat of vaporization and the boiling point. Results gave the following order: $n$-hexane $>2$-MeTHF $>$ ethyl acetate $>$ CPME $>$ DMC $>$ IPA. That means that $n$-hexane requires less $E$ to be evaporated than all the evaluated green solvents. Nonetheless, 2-MeTHF and CPME require only 4 and 9\% more $E$, respectively. Moreover, both solvents extract more carotenes under the same operation conditions than $n$-hexane, which means that economic cost generated by the extra $E$ can be compensated by the higher carotenoid extraction yield. In addition the mass $\mathrm{CO}_{2}$ generated by $\mathrm{kg}$ of solvent is directly related to $E$; therefore its behavior is the same shown by $E$. On the other hand, CPME and 2-MeTHF are two solvents that in the last years are being increasingly used within the academic and industrial chemical communities. This is due to the fact that they offer better physical and chemical properties, as well as lower toxicity ${ }^{\mathbf{1 9 , 2 5}}$ when compared with solvents derived from fossil sources. So, this makes these solvents a proper alternative to extract natural products especially for the food industry.

Table 5 Technical and ecological parameters of solvent used experimentally ${ }^{a}$

\begin{tabular}{|c|c|c|c|c|c|c|c|}
\hline Solvent & $\begin{array}{l}\text { Boiling point } \\
\left({ }^{\circ} \mathrm{C}\right)\end{array}$ & Resource & Toxicity index & $\log P$ & $\begin{array}{l}\text { Energy evaporation of } \\
1 \mathrm{~kg} \text { solvent }(E)(\mathrm{kW} \mathrm{h})\end{array}$ & $\begin{array}{l}E \text { compared } \\
\text { to hexane }(\%)\end{array}$ & $\begin{array}{l}\text { Mass } \mathrm{CO}_{2} \text { generated } \\
\text { by kg of solvent }(\mathrm{g})\end{array}$ \\
\hline n-Hexane & 68.5 & Petroleum & 5 & 3.94 & 0.121 & - & 96 \\
\hline CPME & 105.3 & Chemical synthesis & 4 & 1.41 & 0.132 & +9 & 106 \\
\hline 2-МеTHF & 79.9 & Cereal crop & 4 & 0.82 & 0.126 & +4 & 101 \\
\hline Ethyl acetate & 73.9 & Cereal crop & 5 & 0.71 & 0.127 & +5 & 102 \\
\hline DMC & 90.5 & Chemical synthesis & 5 & 0.15 & 0.194 & +60 & 155 \\
\hline IPA & 73.0 & Cereal crop & 5 & 0.16 & 0.219 & +81 & 175 \\
\hline
\end{tabular}

${ }^{a}$ The values of the solvent parameters in the table were obtained from: https://ilab.acdlabs.com/ilab2/. 


\section{Conclusions}

The aim of this study was evaluate the potential of various green, non-toxic and biodegradable solvents to replace the $n$-hexane for the extraction of the main carotenoids from carrots. The alternative solvents used in the experimental work were selected taking into account the results obtained from HSP and COSMO-RS simulations as well as their theoretical parameters. In the experimental study CPME and 2-MeTHF showed higher extraction yield for $\alpha$-carotene and $\beta$-carotene while ethyl acetate was similar to $n$-hexane. That means that those solvents are promising green solvents to replace $n$-hexane due to their properties to dissolve the carotenoids in carrots and their low toxicity, which is very important for the food industry.

\section{Acknowledgements}

This work was supported in part thanks to funding from the fellowship given to Edinson Yara Varón (no. TECSPR14-2-0029) from the People Programme (Marie Curie Actions) of the Seventh Framework Programme of the European Union (FP7/ 2007-2013) under REA grant agreement no. 600388 (TECNIOspring programme), and from the Agency for Business Competitiveness of the Government of Catalonia, ACCIÓ. The authors also are grateful to the Avignon University and Lleida University for the financial and academic support.

\section{References}

1 A. Aberoumand, World J. Dairy Food Sci., 2011, 6, 71-78.

2 BCC Research LLC, The global market for carotenoids, report code: FOD025E, Wellesley, MA, USA, 2015.

3 J. Berman, U. Zorrilla-López, G. Farré, C. Zhu, G. Sandmann, R. M. Twyman, T. Capell and P. Christou, Phytochem. Rev., 2015, 14, 727-743.

4 I. Jaswir, D. Noviendri, R. F. Hasrini and F. Octavianti, J. Med. Plants Res., 2011, 5, 7119-7131.

5 B. K. Ishida and M. H. Chapman, J. Agric. Food Chem., 2009, 57, 1051-1059.

6 A. G. Sicaire, M. Vian, F. Fine, F. Joffre, P. Carre, S. Tostain and F. Chemat, Int. J. Mol. Sci., 2015, 16, 8430-8453.

7 P. T. Anastas and J. C. Warner, Green chemistry: theory and practice, Oxford Science, New York, 1998.
8 C. Estévez, in Sustainable Solutions for Modern Economies, ed. R. Höfer, RSC Publishing, Cambridge, 2009, ch. 10, pp. 407423.

9 L. Moity, M. Duran, A. Benazzouz, V. Molinier and J. M. Aubry, Alternative Solvents for Natural Products Extraction, ed. F. Chemat and M. A. Vian, Springer-Verlag, Berlin, Heidelberg, 2014, ch. 1, pp. 1-24.

10 C. D. Tanzi, M. A. Vian, C. Ginies, M. Elmaataoui and F. Chemat, Molecules, 2012, 17, 8196-8205.

11 K. Bundeesomchok, A. Filly, N. Rakotomanomana, P. Panichayupakaranant and F. Chemat, LWT-Food Sci. Technol., 2016, 65, 297-303.

12 A. Filly, A. S. Fabiano-Tixier, X. Fernandez and F. Chemat, LWT-Food Sci. Technol., 2015, 61, 33-40.

13 A. Filly, A. S. Fabiano-Tixier, Y. Lemasson, C. Roy, X. Fernandez and F. Chemat, C. R. Chim., 2014, 17, 12681275.

14 Y. Li, A. S. Fabiano-Tixier, C. Ginies and F. Chemat, LWTFood Sci. Technol., 2014, 59, 724-731.

15 C. M. Hansen, Ind. Eng. Chem. Prod. Res. Dev., 1969, 8, 2-11. 16 A. Klamt, Fluid Phase Equilib., 2003, 206, 223-235.

17 I. F. Strati and V. Oreopoulou, Food Chem., 2011, 129, 747752.

18 K. Watanabe, N. Yamagiwa and Y. Torisawa, Org. Process Res. Dev., 2007, 11, 251-258.

19 K. Watanabe, Molecules, 2013, 18, 3183-3194.

20 A. G. Sicaire, M. A. Vian, A. Filly, Y. Li, A. Bily and F. Chemat, Alternative Solvents for Natural Products Extraction, ed. F. Chemat and M. A. Vian, Springer-Verlag, Berlin, Heidelberg, 2014, ch. 12, pp. 253-268.

21 M. Fikselová, S. Silhár, J. Marecek and H. Francaková, Czech J. Food Sci., 2008, 26, 268-274.

22 A. S. Amosova, A. D. Ivakhnov, T. E. Skrebets, N. V. Ulyanovskiy and K. G. Bogolitsyn, Russ. J. Phys. Chem. $B, 2014$, 8, 963-966.

23 T. Grauwet, B. T. Kebede, R. M. Delgado, L. Lemmens, F. Manzoni, L. Vervoort, M. Hendrickx, J. S. Elmore and A. V. Loey, Eur. Food Res. Technol., 2015, 240, 183-198.

24 J. M. Prado, P. C. Veggi and M. A. Meireles, Curr. Anal. Chem., 2014, 10, 29-66.

25 V. Antonucci, J. Coleman, J. B. Ferry, N. Johnson, M. Mathe, J. P. Scott and J. Xu, Org. Process Res. Dev., 2011, 15, 939-941. 